\title{
WIRELESS VIDEO STREAMING WITH COLLABORATIVE ADMISSION CONTROL FOR HOME NETWORKS
}

\author{
Monchai Lertsutthiwong, Thinh Nguyen, and Alan Fern \\ School of Electrical Engineering and Computer Science \\ Oregon State University, Corvallis, OR 97331, USA \\ \{lertsumo, thinhq, afern\}@eecs.oregonstate.edu
}

\begin{abstract}
Limited bandwidth and high packet loss pose a serious challenge for video streaming over wireless networks. Even when packet loss in the medium is not present, the fluctuating available bandwidth due to varying number of active flows in a network causes problem for video streaming applications. In this paper, we propose to employ a novel admission control together with a ratedistortion optimized framework to maintain reasonable qualities for multiple concurrent video streams. In particular, we formulate an optimization problem to allocate the optimal transmission rates for each layered video streams jointly with the MAC protocol of a slightly modified $802.11 \mathrm{x}$ network. We show the hardness results of the optimization problem under various conditions. Furthermore, we show that a simple greedy layer-allocation algorithm is typically not optimal, although it can approximate the solution reasonably well under certain assumptions.
\end{abstract}

\section{INTRODUCTION}

Recent years have witnessed an explosive growth in multimedia wireless applications such as video streaming and conferencing. One of the reasons for this tremendous growth is the deployment of IEEE 802.11x WLAN in both private homes and enterprise networks. Without a proper bandwidth allocation mechanism in a wireless network, a flow may experience significant performance degradation during a session due to free admission of an arbitrarily large number of flows. Thus, admission control is used to prevent the new flow from joining the network in order to maintain a reasonable quality of the existing flows.

Admission control is typically performed at the edge of a wired network. The decision to admit or reject a new flow is easier to make, compared to that of a wireless network. A simple admission control algorithm for wired networks can keep track of the total used bandwidth. The available bandwidth is then equal to the difference between the network capacity and used bandwidth. A new flow is admitted if its requested bandwidth is smaller than the available bandwidth by some threshold, otherwise it is rejected. On the other hand, admission control for a wireless network is more complex. This complication is due to the channel contention access, in which the interferences, i.e., the collisions between the new flow and the existing flows reduce all the flow's throughputs. The number of these collisions increases nonlinearly with the number of competing flows, making it harder for an admission control algorithm to decide whether or not to admit a new flow. In particular, to decide whether or not to admit a video stream of $K$ kbps, the admission control algorithm must ensure that the available bandwidth is at least $K+H$ kbps where $H$ is the incurred overhead.

This paper proposes a novel collaborative admission control algorithm in a slightly modified $802.11 x$ network. In the proposed admission control, a device that wants to inject a new flow into the network, distributes its video profile to all other nodes. Any of the existing devices in the network voluntarily performs the admission control operation and determines whether a new flow should be injected or not based on the requirements of other flows. In particular, we consider the problem of optimizing the qualities of multiple layered video streams [1][2] simultaneously in a wireless home network. When there is not enough bandwidth in the network to accommodate all the video streams at their full rates (all the layers), each video sender voluntarily reduces the number of layers [3], and thus increases the distortion. The optimization problem would be to select which layers of which streams to be dropped in such a way to maximize the weighted sum of visual quality for all the videos. We will show that this optimization problem is in general, NP-hard due to the overhead $H$ and the rate distortion models of different video streams.

The outline of our paper is as follows. In section 2, we describe our proposed collaborative admission control algorithm, and show how to compute the overhead $H$. We then formulate the optimization problem in Section 3 and report the computational complexities in Section 4. Next, we show experimental results in Section 5, and provide few concluding remarks in Section 6.

\section{COLLABORATIVE ADMISSION CONTROL}

Many admission control algorithms [4] have been proposed for $802.11 x$ network. In this section, we describe a novel collaborative admission control for $802.11 \mathrm{x}$ like network. While our algorithm can be implemented at the access point, we will describe it in the context of ad-hoc networks, which eliminate the need for an access point [5].

We first briefly describe the MAC protocol of $802.11 \mathrm{x}$ based network. Contention based access enables multiple devices to compete for a shared wireless channel. While there are many parameters in $802.11 \mathrm{x}$ standards, for simplicity, we focus our discussion on the contention window $(\mathrm{CW})$. To access the channel, a device first senses the channel. If the channel is idle, the device starts sending the data. Otherwise, it sets a back-off timer for a random number of time slots between $[0, C W]$. The back-off timer is decremented by one for each idle time slot, and halts decrementing when a transmission is detected. The decrementing 
resumes when the channel is sensed idle again. A device can begin transmission on the channel as soon as its back-off timer reaches zero. If a collision occurs, $C W$ is doubled for each retransmission in order to reduce the traffic in a heavily loaded network.

In our proposed modification, $C W$ is not doubled after a loss. We argue that doubling $C W$ is not optimal when a proper admission control is used. Using admission control, the traffic load at any given time is known or highly predictable, and therefore $C W$ of each device can be precisely tuned (by the admission control algorithm) to achieve the required throughput. For ease of analysis, we will use the transmission probability $p=1 / \mathrm{CW}$ to control the sending rate of a device. Higher $p$ results in high likelihood of sending a packet thus, resulting in higher rate.

We also use an RTS/CTS packet to reserve the requested bandwidth before sending a data packet. Once the channel is successfully reserved, we assume that the data packet is successfully sent. Suppose a devices wants to start a new flow of throughput $x$ kbps. It first distributes its video-distortion profile to all other nodes. It is important to note that all the admission control operations are voluntarily done by any of the existing devices in the network because each node knows all the video profiles from everyone. Once the node responsible for performing the admission control receives this new video-distortion profile, it will run the rate-distortion optimization and see whether there is a solution to accept a new flow or not. If a solution exists, then this device will broadcast the updated transmission parameters $p$ 's to other devices. This approach reduces the workload for other devices.

We consider breaking the wireless channel into time slots that are shared among the nodes. There are three types of slots: I-type, $C$-type, and S-type slots. Each individual slot for all types has the same length. Each node regularly monitors all traffic and estimates the following parameters:

- $\quad I$ : percentage of idle slots (I-type slots)

- $\quad C$ : percentage of collision slots (C-type slots)

- $\quad S_{i}$ : percentage of slots with a successful transmission of $R T S / C T S$ packets (S-type slots) for flow $i$

- $S$ : percentage of slots with a successful transmission of $R T S / C T S$ packets for all the flows

The amount of allocated slots for an RTS/CTS packet depends on its length. The relationship between $C, S_{i}, S$, and $I$ is shown in (1)

$$
S=\sum_{i} S_{i}, I+C+\sum_{i} S_{i}=1
$$

Suppose we are going to inject a new flow $j$ with the transmission probability $p_{j}$. Then there is no impact for the $C$-type slots but the collision may occur in $S$-type slots with probability $p_{j}$. For I-type slots, it would become $S$-type slots with probability $p_{j}$. All updated parameters can be computed by using their current values.

$$
\begin{aligned}
& I_{\text {new }}=I_{\text {current }}\left(1-p_{j}\right) \\
& C_{\text {new }}=C_{\text {current }}+S_{\text {current }} p_{j} \\
& S_{i, \text { new }}=S_{i, \text { current }}\left(1-p_{j}\right) \\
& S_{\text {new }}=S_{\text {current }}\left(1-p_{j}\right)+I_{\text {current }} p_{j} \\
& S_{j}=I_{\text {current }} p_{j}
\end{aligned}
$$

We can also represent the above expression in terms of the transmission probability for all the flows as (7),

$$
I_{\text {new }}=\prod_{\forall i}\left(1-p_{i}\right), \quad S_{i, \text { new }}=p_{i} \prod_{\substack{k \subset n \\ k \neq i}}\left(1-p_{k}\right)
$$

where $n$ is the numbers of the flows. In our technical report [6], we show how to use Equations (2)-(7) to compute appropriate $p_{i}$ and the collision overhead $H$ given a set of required rates, which are proportional to $S_{i}$ 's.

\section{PROBLEM FORMULATION}

We are now at the position to formulate a rate-distortion optimization problem for multiple layered video streams under bandwidth constraint. We note that the average throughput per unit time or transmission rate $R_{i}$ for flow $i$ can be achieved by setting its transmission probability $p_{i}$. When there is enough bandwidth for everyone, $p_{i}$ 's are set to large values so that all the layers of all the video streams would be sent. When there is not enough bandwidth, e.g. due to too many flows, the layers from certain videos are dropped to result in the least average distortions over all the videos.

For a simple scenario, we assume that there is no packet loss during transmission over the channel. The transmission rate $R_{i}$ for flow $i$ depends on the number of successfully transmitted RTS/CTS packets $K_{i}$, proportional to the number of transmitted video layers $l_{i}$. Large video layer may be split up into multiple packets. The maximum size of each packet for flow $i$ is equal to the transmission opportunity $T X O P_{i}$.

$$
R_{i}\left(l_{i}\right)=K_{i} T X O P_{i}
$$

The optimization problem studied in this paper is to select the optimal number of video layers to transmit for each of the $n$ nodes (streams). The problem is specified by giving: for each node, a function $D_{i}\left(l_{i}\right)$, that gives the reduction in distortion when using $l_{i}$ layers at node $i$; a rate function $R_{i}\left(l_{i}\right)$, that gives the required bandwidth for transmitting $l_{i}$ layers from node $i$; an overhead function $H\left(l_{l}, \ldots, l_{n}\right)$, that gives the amount of bandwidth consumed by overhead (e.g. due to channel contention) for a given assignment of layers to nodes; lower bounds on the distortion for each $i$ denoted by $L_{i}$; and finally a bound on the total bandwidth $B W$. Given these quantities, the optimization problem is as follows:

$$
\begin{array}{ll}
\text { maximize } & \sum_{i=1}^{n} D_{i}\left(l_{i}\right) \\
\text { over } & l_{i} \\
\text { subject to } & D_{i}\left(l_{i}\right) \geq L_{i} \\
& \sum_{i=1}^{n} R_{i}\left(l_{i}\right)+H\left(l_{1} \ldots, l_{n}\right) \leq B W
\end{array}
$$

That is, we must find the assignment of layers to each node that maximizes the reduction in total distortion subject to bandwidth and local minimum distortion constraints.

\section{COMPUTATIONAL COMPLEXITY}

Our optimization problem is distinct from most other bandwidth optimization problems by its inclusion of the overhead term $H$ in the bandwidth constraint. Thus, existing algorithms and complexity proofs do not apply to our problem. Here we analyze the computational complexity of problem classes with the form given in the previous section. We begin by stating three assumptions about the optimization problem and consider the complexity under various subsets of these assumptions according to significant quantities in layered video encoding techniques. 
Assumption 1: Uniform rate increase per level

$$
R_{i}\left(l_{i}+1\right)-R_{i}\left(l_{i}\right)=R_{j}\left(l_{j}+1\right)-R_{j}\left(l_{j}\right)
$$

Assumption 2: Diminishing returns

$$
D_{i}\left(l_{i}+1\right)-D_{i}\left(l_{i}\right)<D_{i}\left(l_{i}\right)-D_{i}\left(l_{i}-1\right)
$$

Assumption 3: Invariant overhead

$$
H\left(\ldots, l_{i}+1, \ldots\right)=H\left(\ldots, l_{j}+1, \ldots\right) \text { for any } l_{l}, \ldots, l_{n}
$$

Below we will also refer to the property of additive overhead which means that $H\left(l_{1}, \ldots, l_{n}\right)$ can be factored as a sum of individual overhead function $H_{i}$. That is,

$$
H\left(l_{1}, \ldots, l_{n}\right)=\sum_{i=1}^{n} H_{i}\left(l_{i}\right)
$$

Our first result is that given all of the above assumptions, we can solve the optimization problem using a greedy layer-allocation algorithm. This algorithm is iterative and simply increments the layer count of one node each iteration, choosing the node that results in the most reduction in distortion without violating the bandwidth constraints. Due to space constraints the reader is referred to the full technical report for proofs of these results.

Proposition 1: The class of problems for which Assumptions 1, 2, and 3 hold can be solved optimally and efficiently using the greedy layer-allocation algorithm.

We now show that in a sense the above assumptions are necessary for the greedy algorithm to be optimal, and furthermore for there to exist any efficient solution algorithm. In particular, the next series of propositions shows that if we remove any one of the assumptions the problem becomes NP-hard. Below our results concern the decision-problem version of the above optimization problem. That is, the problem of deciding whether there is a feasible solution given a particular distortion threshold as input. Note that if the optimization problem can be solved efficiently then so can the decision problem. Thus, hardness results about the decision problem pertain to optimization as well. We note that the proofs of the below hardness propositions are based on various reductions from the knapsack problem.

Proposition 2: The class of decision problems for which Assumptions 2 and 3 hold but Assumption 1 does not is NPcomplete even if we restrict the overhead to be the constant zero function.

This shows that even with a trivial overhead function, Assumption 1 is required to retain tractability of optimization.

Proposition 3: The class of problems for which Assumptions 1 and 3 hold but Assumption 2 does not is NP-complete even if we restrict the overhead to be the constant zero function.

This shows that even with a trivial overhead function, Assumption 2 is required to retain tractability of optimization. Finally, we show that Assumption 3 is also necessary in some sense. In particular, when it is lifted the problem becomes computationally hard even when restricted to the class of problems with additive overhead.

Proposition 4: The class of problems for which Assumptions 1 and 2 hold but Assumption 3 does not is NP-complete even if we restrict to additive overhead.
Table 1. The example of standard video profiles.

\begin{tabular}{c|c|c|c|c|c|c|c|c|c}
\hline \multirow{2}{*}{\begin{tabular}{c} 
Layer \\
\cline { 2 - 10 }
\end{tabular}} & $\begin{array}{c}\text { Bit } \\
\text { Rates } \\
\text { (kbps) }\end{array}$ & $\begin{array}{c}\text { Distortion } \\
\text { (MSE) }\end{array}$ & $\begin{array}{c}\text { Reduced } \\
\text { Distortion } \\
\text { (MSE) }\end{array}$ & $\begin{array}{c}\text { Bit } \\
\text { Rates } \\
\text { (kbps) }\end{array}$ & $\begin{array}{c}\text { Distortion } \\
\text { (MSE) }\end{array}$ & $\begin{array}{c}\text { Reduced } \\
\text { Distortion } \\
\text { (MSE) }\end{array}$ & $\begin{array}{c}\text { Bit } \\
\text { Rates } \\
(\mathbf{k b p s )}\end{array}$ & $\begin{array}{c}\text { Distortion } \\
\text { (MSE) }\end{array}$ & $\begin{array}{c}\text { Reduced } \\
\text { Distortion } \\
\text { (MSE) }\end{array}$ \\
\hline 1 & 64 & - & - & 32 & 123.90 & - & 64 & - & - \\
\hline 2 & 128 & 83.77 & - & 112 & 103.06 & 20.84 & 128 & 71.30 & - \\
\hline 3 & 192 & 63.54 & 20.23 & 160 & 87.72 & 15.34 & 192 & 56.63 & 14.67 \\
\hline 4 & 256 & 50.48 & 13.06 & 208 & 78.18 & 9.54 & 256 & 46.03 & 10.60 \\
\hline 5 & 320 & 38.29 & 12.19 & 256 & 71.30 & 6.88 & 320 & 39.18 & 6.85 \\
\hline 6 & 384 & 32.59 & 5.70 & 304 & 65.03 & 6.27 & 384 & 33.35 & 5.83 \\
\hline 7 & 448 & 27.74 & 4.85 & 352 & 57.95 & 7.08 & 448 & 29.05 & 4.30 \\
\hline 8 & 512 & 23.61 & 4.13 & 400 & 51.65 & 6.30 & 512 & 25.89 & 3.16 \\
\hline
\end{tabular}

These complexity results show that if we remove any one of the three assumptions the problem becomes NP-hard and hence is not likely to be solved by an efficient algorithm, in particular the greedy algorithm. The results also show that this is true even if we place strict restrictions on the form of the overhead function. In particular, even if the overhead is additive the problem is hard as shown by Proposition 4.

Unfortunately we can show that the overhead function arising in our protocol is not invariant as required by Assumption 3.

Proposition 5: The overhead of the CDAC algorithm is not overhead invariant.

This shows that the greedy algorithm is not likely to be optimal for our particular problem. The results motivate investigating whether there are variants of the protocol that are overhead invariant to allow for tractable optimization in the presence of Assumptions 1 and 2 , which will often be satisfied in practice.

\section{SIMULATION RESULTS}

We use three standard video profiles in Table 1 for flow 1, flow 2, and flow 3, which are AKIYO, COASTGUARD, and FOREMAN [3][7]. The sizes of RTS/CTS packets, maximum bandwidth, and TXOP are 64 bytes, $1 \mathrm{Mbps}$, and 1500 bytes, respectively. To be fair among all flows, they use the same TXOP.

Fig. 1(a) shows the results from optimal solution based on Table 1. In order to guarantee the acceptable quality of each video streaming, the transmission rate initially starts at the assumed minimum rate of $256 \mathrm{kbps}, 208 \mathrm{kbps}$, and $192 \mathrm{kbps}$ for flow 1 , flow 2 , and flow 3 , respectively. We compare the throughput of each flow to the normalized total bandwidth usage, which is the ratio between used bandwidth and total bandwidth $B W$. Total bandwidth usage composes of throughput, overhead S-type slots, and overhead C-type slots. The optimal solution determines the combination of all the flows that achieves the minimum distortion under the constraint in (9). The transmission probability for each flow keeps increasing in order to achieve the requested bandwidth as shown in Fig. 1(b). Based on (10), the flow with higher number of layers will have higher rate of transmission probability than other flows. The percentage of $S$-type slots is proportional to the transmission probability as shown in Fig. 1(c). Furthermore, the percentage of I-type, C-type, and S-type slots is shown in Fig. 1(d). Fig. 1(e) shows the change in distortion when we add a new layer into the existing flows. When the total throughput is increasing, the overall distortion will be diminishing as Fig. 1(f).

We compare the results between different algorithms: optimal solution, greedy algorithm, and other basic strategies (e.g. fairly limit the maximum throughput of each flow) as Fig. 2 and Table 2. 


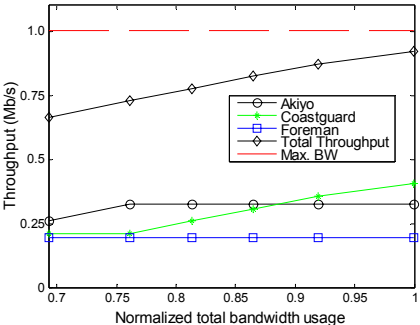

(a)

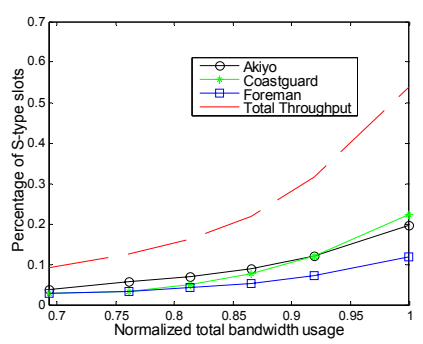

(c)

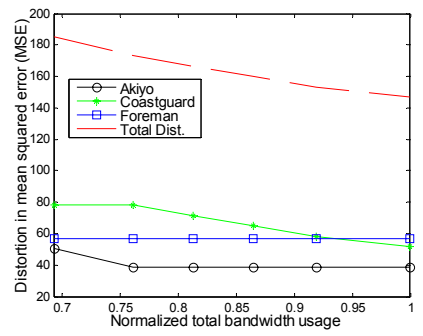

(e)

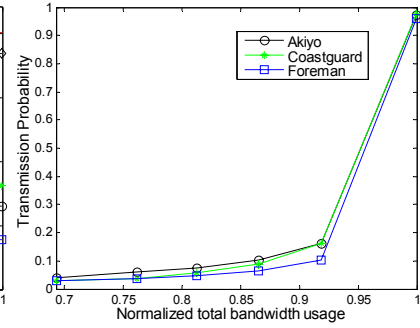

(b)

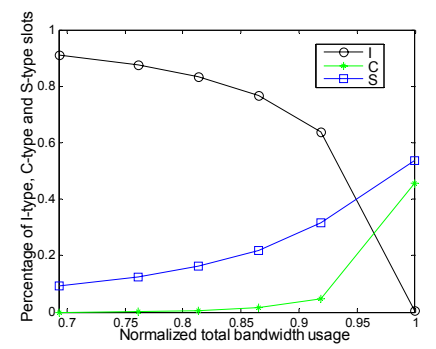

(d)

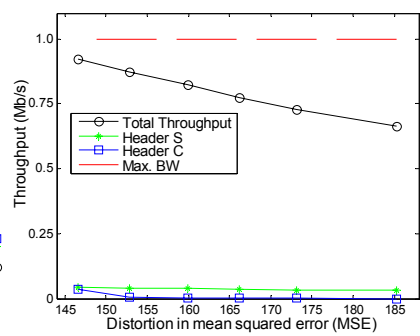

(f)
Fig. 1. The results for optimal solution over three video flows (a) Throughput. (b) Transmission probability. (c) Percentage of $S$ type slots. (d) Percentage of $I$-, $C$ - and S-type slots. (e) Distortion in MSE. (f) Rate-distortion characteristic.

Optimal solution achieving the minimum overall distortion implies that the selection of all flows is the best combination. Because standard profiles in Table 1 are not uniform, the result from greedy algorithm would not be optimal.

Based on greedy algorithm, the effect of changing the size of TXOP and RTS/CTS portions is shown in Table 3. If we increase the size of TXOP but the size of RTS/CTS and the overall distortion remain the same, a number of transmitted packets are reduced. This causes a smaller portion of overhead in the network and the transmission probability would decrease. We use this relationship to determine the appropriate size of TXOP and RTS/CTS to enhance the bandwidth efficiency. Simulation results demonstrate that our proposed algorithm improves the video quality up to $26 \%$ as compared to those resulted from transmissions in a typical 802.11x-based network [8].

\section{CONCLUSIONS}

In this paper, we have proposed to employ a novel admission control together with a rate-distortion optimized framework to maintain reasonable qualities for multiple concurrent video streams. In particular, we formulated an optimization problem to allocate the optimal transmission rates for each layered video streams jointly with the MAC protocol of a slightly modified
Table 2. Compare the results from different algorithms.

\begin{tabular}{c|c|c|c|c|c|c|c|c}
\hline & \multirow{2}{*}{$\begin{array}{c}\text { Distortion } \\
\text { (MSE) }\end{array}$} & \multicolumn{2}{|c|}{ Normalized Bandwidth Usage (Percent) } & \multicolumn{3}{|c}{ Transmission Probability } \\
\cline { 3 - 9 } & & Total & $\begin{array}{c}\text { Overhead } \\
\text { S }\end{array}$ & $\begin{array}{c}\text { Overhead } \\
\text { C }\end{array}$ & Throughput & AKIYO & $\begin{array}{c}\text { COAST- } \\
\text { GUARD }\end{array}$ & FOREMAN \\
\hline $\begin{array}{c}\text { Optimal } \\
\text { Solution }\end{array}$ & 146.57 & 99.96 & 4.24 & 3.60 & 92.12 & 0.9752 & 0.9781 & 0.9594 \\
\hline $\begin{array}{c}\text { Greedy } \\
\text { Algorithm }\end{array}$ & 148.77 & 96.59 & 4.24 & 1.84 & 90.51 & 0.3125 & 0.2500 & 0.3125 \\
\hline Others & 149.35 & 93.93 & 4.14 & 0.90 & 88.89 & 0.2036 & 0.1814 & 0.1698 \\
\hline
\end{tabular}

Table 3. The effects of changing the size of TXOP and RTS/CTS.

\begin{tabular}{c|c|c|c|c|c|c|c|c|c}
\hline \multirow{2}{*}{$\begin{array}{c}\text { TXOP } \\
\text { (Bytes) }\end{array}$} & \multirow{2}{*}{$\begin{array}{c}\text { RTS/CTS } \\
\text { (Bytes) }\end{array}$} & Distortion & \multicolumn{3}{c|}{ Normalized Bandwidth Usage (Percent) } & \multicolumn{2}{c}{ Transmission Probability } \\
\cline { 5 - 11 } & & & Total & $\begin{array}{c}\text { Overhead } \\
\text { S }\end{array}$ & $\begin{array}{l}\text { Overhead } \\
\text { C }\end{array}$ & Throughput & AKIYO & $\begin{array}{c}\text { COAST- } \\
\text { GUARD }\end{array}$ & FOREMAN \\
\hline 1300 & 64 & 148.77 & 99.96 & 5.02 & 4.43 & 90.51 & 0.9744 & 0.9671 & 0.9744 \\
\hline 1300 & 150 & 162.50 & 99.89 & 10.30 & 10.40 & 79.19 & 0.9761 & 0.9625 & 0.9703 \\
\hline 1300 & 300 & 185.29 & 99.83 & 17.21 & 16.36 & 66.26 & 0.9758 & 0.9693 & 0.9680 \\
\hline 1500 & 64 & 148.77 & 96.59 & 4.24 & 1.84 & 90.51 & 0.3125 & 0.2500 & 0.3125 \\
\hline 1500 & 150 & 155.62 & 99.92 & 9.21 & 6.67 & 84.04 & 0.9785 & 0.9709 & 0.9733 \\
\hline 1500 & 300 & 173.10 & 99.87 & 16.00 & 11.14 & 72.73 & 0.9816 & 0.9697 & 0.9697 \\
\hline
\end{tabular}

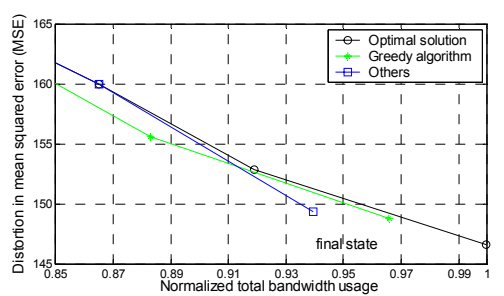

Fig. 2. Compare the results of overall distortion.

$802.11 x$ network. We show the hardness results of the optimization problem under various conditions. Furthermore, we show that a simple greedy layer-allocation algorithm is typically not optimal, although it can approximate the solution reasonably well under certain assumptions.

\section{REFERENCES}

[1] W. Li, "Overview of fine granularity scalability in MPEG-4 video standard", IEEE trans. on circuits and systems for video tech, vol. 11, no. 3, pp.301-316, Mar. 2001

[2] L. P. Kondi, F. Ishtiaq and A. K. Katsaggelos, "On video SNR scalability", IEEE Int. conf. on image processing, vol. 3, pp. 934-938, Oct. 1998

[3] J. Chakareski and P. Frossad, "Rate-distortion optimized packet scheduling of multiple video streams over shared communication resources", IEEE trans. on multimedia, vol. 8, no. 2, pp. 207-218, Apr. 2006

[4] D. Gao, J. Cai and K. N. Ngan, "Admission control in IEEE 802.11e wireless LANs", IEEE network, vol. 19, pp. 6-13, Jul.-Aug. 2005

[5] E. Setton, T. Yoo, X. Zhu, A. Goldsmith and B. Girod, "Cross-layer design of ad hoc networks for real-time video streaming", IEEE wireless comm., pp. 59-65, Aug. 2005

[6] M. Lertsutthiwong, T. Nguyen, and A. Fern, "Wireless video streaming with collaborative admission control for home networks", Technical report, ECE07-06-01, Oregon State University, Apr. 2007

[7] X. Sun, F. Wu, S. Li, W. Gao and Y. Zhang, "Macroblock-based progressive fine granularity scalable (PFGS) video coding with flexible temporal-SNR scalabilities", IEEE Int. conf. on image processing, vol. 2, pp. 1025-1028, Oct. 2001

[8] G. Bianchi, "Performance analysis of the IEEE 802.11 distributed coordination function", IEEE journal in comm., vol. 18, no. 3, pp. 535-547, Mar. 2000 